\section{PREVALENCE AND RISK FACTORS OF DRY EYE AMONG PATIENTS OF A TERTIARY CARE CENTRE IN EASTERN INDIA}

KEY WORDS:Dry eye, McMonnies questionnaire Schirmer's test, Tear film breakup time.
Dr. Nilesh Mohan

\section{Dr. Vandana Parasar}

\section{Dr. Ankita Singh}

Dr. Rakhi

Kusumesh*
MD (AIIMS), DNB, ICO (Basic Ophtha) Additional Professor, RIO, IGIMS, Sheikhpura, Patna

MS Senior Resident, RIO, IGIMS, Sheikhpura, Patna

AIM: To determine the prevalence and risk factors of dry eye among patients of a tertiary care centre in eastern India. Symptoms of dry eye are encountered as one of the most frequent complains among the patients attending the outpatient department in ophthalmology commonly presenting as ocular discomfort, burning sensation and foreign body sensation. Prevalence of this entity is still not known in our study population due to lack of specificity of symptoms and diagnostic criteria. MATERIALS AND METHODS: A prospective, observational, cross-sectional study was conducted among patients attending ophthalmology OPD in a tertiary care teaching hospital of Bihar. $4116(16.64 \%)$ patients with dry eye symptoms were examined after taking informed consent. RESULTS: 1620 (6.55\%) patients were found to have dry eye based on McMonnies questionnaire, Schirmer's test and tear film breakup time. There were 1180 female and 440 male with dry eye. Male to female ratio was 2.7:1.Non-tribals were affected more commonly than tribals. Students, outdoor workers and office worker with professional constituted over fifty percent of dry eye cases. CONCLUSION: Prevalence of dry eye was more in females and elderly as compared to male and younger population respectively. Burning sensation and ocular discomfort was the most common presenting complains.

\section{INTRODUCTION:}

Burning sensation and ocular irritation is the common presenting complains in patient attending ophthalmology OPD during summer. The condition is diagnosed as dry eye, a disorder of tear films. Keratoconjunctivitis sicca (KCS) is a multifactorial disease caused by decreased tear production or excessive tear evaporation, leading to the symptoms of dry eyes ${ }^{1}$.

Normal tear film consists of three layers: superficial lipid layer produced by the meibomian glands, middle aqueous layer produced by the lacrimal gland and accessory lacrimal glands of Wolfring and Krause and innermost mucin layer produced by the goblet cells. Each layer has their own specific functions. Lipid layer prevents evaporation of tear films, middle layer constitutes $90 \%$ of ear film and inner layer lowers the surface tension and renders the corneal surface wet $^{2}$.

There are two main categories of dry eye conditions. Aqueous tear-deficient dry eye (ATDDE), caused by deficiency of lacrimal gland in producing tears e.g. Sjogren's syndrome (SS) Evaporative dry eye (EDE) caused by excessive evaporation of tears e.g. dry eye due to meibomian gland dysfunction, ocular surface disorders, low blink rate, computer vision syndrome etc. In diabetes mellitus, lacrimal gland dysfunction, decreased corneal sensitivity and loss of goblet cells leads to dry eye syndrome. Various drugs and disease conditions also can lead to dry eyes like symptoms ${ }^{3}$.

Early diagnosis is the key in preventing patients from visionthreatening complications of dry eye disease. Symptom assessment with screening and diagnostic tests are used to establish the diagnosis of dry eye. McMonnies questionnaire is a well-balanced and focused screening test for dry eye $e^{4}$.It is a subjective test with sensitivity of $87-98 \%$ and specificity $87-$ $97 \%{ }^{4}$. This questionnaire consists of 12 questions related to the risk factors for DED. These include demographic information, dry eye symptoms, previous and current dry eye treatments, secondary symptoms (associated with environmental stimuli), systemic conditions (Sjögren syndrome, arthritis, thyroid disease), and dryness of the mucous membranes (chest, throat, mouth or vagina) ${ }^{5}$. Due to subjective nature of self-reported symptoms, test is reliable and repeatable than objective clinical tests in detecting dry eye. A study done by Nichols KK showed that objective test such as Schirmer's test adds to the final diagnosis of dry eye disease ${ }^{6}$. Tear film breakup time (TBUT) is a clinical test used to assess evaporative dry eye disease. To measure TBUT, tear film is stained with sodium fluorescein $1 \%$ and patient is asked not to blink while the tear film is observed under a broad beam of cobalt blue illumination in slit lamp. Time is noted for the first appearance of a "dark" dry spot which is recorded as the TBUT ${ }^{7}$. Thus, this study used McMonnies questionnaire for screening and Schirmer's test and TBUT for diagnosis of dry eye disease to evaluate the proportion of dry eye cases among medical students of a tertiary Care hospital ${ }^{8}$.

AIM: To determine the prevalence and risk factors of dry eye among patients of a tertiary care centre in eastern India.

MATERIALS AND METHODS : This was a cross-sectional study conducted in the Regional Institute of Ophthalmology, Indira Gandhi Institute of Medical Sciences, Sheikhpura ,Patna between August 2018 to September 2019 after taking approval of the competent authority.Study was done in accordance with the principles expressed in the Declaration of Helsinki. 4116 patients attending the eye OPD and provisionally diagnosed with dry eye disease were selected for the study meeting the inclusion criteria. Informed consent form written in English and vernacular languages were obtained from each participant. Collection and analysis of the data were anonymous; In addition, the clinical tests did not cause any physical harm to patients. We believed nothing was against health, safety and privacy of patients in this study. Inclusion criteria: Patients attending ophthalmology OPD with age at presentation above 18 years with presenting complain consistent with dry eyes (ocular discomfort, burning sensation, redness, itching, dryness etc) were included in this study.

Exclusion criteria: Patients taking any ocular or systemic 
medications, or had undergone intra or extra ocular surgery or with an active ocular infection in the previous 6 months were excluded from the study also patient not giving consent or had not agreement with the study were excluded. Procedures for assessing the dry eye-Subjects were asked to fill McMonnies questionnaire which had 14 questions that focused on the clinical risk factors for dry eye. These were related to age, gender, dry eye symptoms (foreign body sensation, burning, itching etc ) feeling of dryness and tiredness, previous treatment for dry eye, secondary symptoms (associated with environmental stimuli), systemic diseases, dryness of mucous membranes, and systemic medications ${ }^{10}$. Scoring of the dry eye-The scores ranged from 0 to 45. Individuals having McMonnies score greater than 14.5 were evaluated for Dry Eye diagnosis ${ }^{11}$. Schirmer's test $-\mathrm{A}$ sterilized Schirmer's strip (5 X $35 \mathrm{~mm}$ Whatman filter paper no. 41) was placed over the junction of medial two- third and lateral one-third of lower lid after instilling a drop of $(0.5 \%)$ Proparacaine. The test was considered positive when the the level of strip wetting (in millimeters) after five minutes was less than ten millimeters. Positive Schirmer's test represents aqueous tear deficiency ${ }^{10}$. Procedure of tear film break-up timeA sterilized $2 \%$ fluoroscein strip was moistened with lubricating eye drop and placed in the lateral one-third of lower lid in a non-anaesthetised eye and patient was asked to blink only once or twice to avoid pooling of fluorescein, following which the strip was removed. Tear film was observed on Cobalt blue light of the slit lamp and time was noted. Time lapse between the last blink and the appearance of the first randomly distributed dark discontinuity in the fluoroscein-stained tear film is the tear break up time.Values of less than 10 seconds were considered abnormal ${ }^{10}$.

Diagnosis of dry eye- It was done with positive Schirmer's test (wetting $<10 \mathrm{~mm}$ ) and TBUT of $<10 \mathrm{~s}$ along with McMonnies score more than $14.5^{11}$. The results of tear function tests were further subjected to scoring system (Khurana (1993) scoring system to assess the severity of dry eye ${ }^{12}$. According to their scores, the patients were graded to be having

1.No dry eye $(0-1)$

2.Dry eye suspect (2)

3. Mild dry eye (3-8)

4. Moderate dry eye (9-13)

5. Severe dry eye (14-18)

Data were noted on a standardized pro forma.

\section{RESULTS :}

During the study period total 24732 patients attended the ophthalmology OPD, of which 4116 (16.64\%) patients were suspected cases of dry eyes. These cases were further examined with McMonnies Questionnaire, Schirmer's test and Tear film breakup time (TBUT) to confirm the diagnosis and classify the disease in mild, moderate and severe dry eyes cases. $1620(6.55 \%)$ subjects had McMonnies score above 14.5 and confirmed to have dry eyes ${ }^{11}$.Thus the incidence of dry eyes in the study population was $6.55 \%$. These were further evaluated by Schirmer test and TBUT and classified in mild, moderate and severe cases. $1180(72.83 \%)$ were female and $440(27.1 \%)$ were male [Table 1]. Female to male ratio was 2.7: 1 Dry eye was more common among urban population and in hypermetropes. Also the incidence was lesser in tribal population. Patients were distributed from 18 to 73 years with majority above 42 years. Mean age of the study population was 49.1 years. Figure 1 represents age distribution of the patients with dry eyes. In all the three groups, dry eye was more common in female than male. In male most common age group affected was 18-40 years while female above 61 years were affected most commonly. Farmers, labourers and outdoor workers were the two occupation groups affected more commonly (Figure 2). Table 2 represents the common symptoms reported by the patients. Most common symptom reported was burning sensation, ocular discomfort and foreign body sensation. In this study, moderate grade of dry eyes was seen in about $61.37 \%$ of cases, while mild and severe grade of dry eyes were seen in 32.60 and $8.38 \%$ of cases respectively.

\section{Table 1}

Population Characteristics of Dry eye cases $(n=405)$

1. Male : Female $=110: 295$

2. Rural : Urban $=191: 214$

3. Tribal $:$ Non tribal $=127: 278$

4. Refractive status

Hypermetropes $=210$

Myopes $=126$

Emmetropes $=69$

\section{Table 2}

Common symptoms reported by patients with dry eye

Burning sensation

Ocular discomfort

Foreign body sensation

Watering

Dryness

Grittiness

Itching

Scratchiness

Redness

Ocular pain

Photophobia

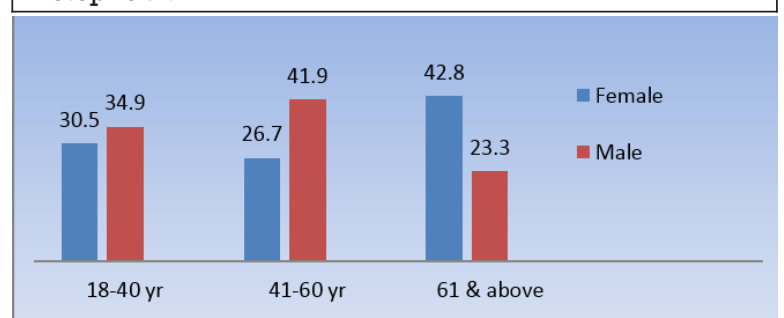

Figure 1 : Age distribution of patients with dry eyes (\%)

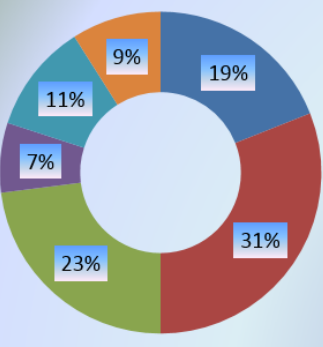

Homemaker/Student

- Farmer/ Labourers

Outdoor workers (High exposure)

Indoor workers (Low exposure)

Figure 2 : Occupation of Dry eye caes

\section{DISCUSSION:}

Dry eye is one of the commonly encountered ocular morbidities seen by ophthalmologists in general practice. It is common in this part of India especially during summers when the climate is dry and hot. The disease is ignored commonly because of nonspecific symptoms and so the exact prevalence of this condition is not estimated precisely. The diagnostic accuracy of various subjective and objective methods varies a lot. The overall incidence of dry eye patients diagnosed in our study is $6.55 \%$ which is much lower than the study done at West Bengal ${ }^{13}$ where it was around $54 \%$ and the study conducted by Bhutia et $\mathrm{al}^{14}$ in Sikkim where it was around $12.7 \%$. Thus the proportion of dry eyes varies in different geographical locality.

In our study, relatively higher numbers of female are affected in all age groups as compared to male with overall female: male ratio $2.7: 1$. This was very similar to other studies where females were affected more than male ${ }^{15}$. Various literatures support this finding. A study from United Kingdom suggests 
that evaporation of tear film is significantly higher in elderly and females because of the thinner and less efficient lipid layer in the tear films which is required to prevent the evaporation of tear films ${ }^{16}$. These findings are in concordance with the previous studies by Shaumberg et al, Moss et al emphasizing higher prevalence of dry eye in women and elderly ${ }^{17,18}$. In this study we have also categorized the study population among tribals and non tribals and found the lesser prevalence of dry eye case in tribals (31.35\%). However no such study has been performed previously to the best of our knowledge, studying the ocular disorders among the tribal population in this region of country. Dry eye cases are also common in occupationally exposed population and subjects using modern gadgets like computer and mobile. This may be due to reduced blinking rate while working on monitor for longer duration ${ }^{19}$. This is also reflected in our study where outdoor workers, students and professional together constituted more than half of the dry eye cases. This was very similar to the study by Anshu et al ${ }^{20}$. In our study, number of dry eye cases in male were more in 18-60 years age group while in females this was more in above 61 years age group. This may be attributed to the outdoor activity of males and effect of post menopausal hormonal changes in female in the particular age group respectively ${ }^{21}$. These associations were also seen and explained in the study by Sahai et al in hospital based population from Jaipur in western part of India ${ }^{22}$.

The most common complaints of dry eye patients are burning, heaviness, stickiness, foreign body sensation, redness, itching, blurred vision, and light sensitivity. In our study, the common complaints were burning sensation, ocular discomfort, foreign body sensation, dryness and grittiness. Similar symptoms were also reported in study by Shah et $\mathrm{al}^{23}$ and Bhutia et $\mathrm{al}^{14}$.We followed the scoring system of Khurana et $\mathrm{al}^{12}$ in our study to classify dry eyes in mild, moderate and severe dry eyes. Here moderate dry eyes cases were way ahead of mild and severe dry eyes cases. This was very similar to the study by Choudhary et $\mathrm{al}^{24}$. Most common etiology for dry eyes in our study was vitamin A deficiency in 38\% cases followed by diabetes mellitus in $21 \%$. Among other causes, computer vision syndrome also contributed in $9 \%$ of cases. Similar etiologies were also mentioned in previous studies except for computer vision syndrome, which is a new entry in the list as the modern gadgets like computer, smart phones and television are used very commonly and leads to dry eyes syndrome ${ }^{25}$.

The procedures used for the diagnosis were safe and no side effects were seen in any of the participant while performing Schirmers test and TBUT. Result from questionare and the tests correlated well in this study.

Limitations of the study: Sample size and duration of study was small in the study thus the study does not necessary represent the result for entire population.

\section{CONCLUSION:}

Though dry eye is a frequently encountered entity for general practitioners and ophthalmologists, but still it is underdiagnosed due to lack of specificity of symptoms. Lack of definitive diagnostic tests and time consuming questioners methods making it more complicated and confusing. Most of the cases are due to occupation related and normal aging process. Proper counseling of the patients regarding the chronic nature disease with long term treatment and preventive measures are necessary to relieve ocular discomfort and ensure patient satisfaction with a better quality of life.

\section{REFERENCES}

1. Gumus K, Cavanagh DH. The role of inflammation and antiinflammation therapies in keratoconjunctivitis sicca. Clinical ophthalmology (Auckland, NZ). 2009;3:57-67.

2. Dartt D, Willcox M. Complexity of the tear film: Importance in homeostasis and dysfunction during disease. Experimental eye research. 2013;117:1-3.
3. Kaštelan S, Tomić M, Salopek-Rabatić J, Novak B. Diagnostic Procedures and Management of Dry Eye. BioMed Research International. 2013;2013:309723.

4. McCarty CA, Bansal AK, Livingston PM, Stanislavsky YL, Taylor HR. The epidemiology of dry eye in Melbourne, Australia. Ophthalmology 1998;105:1114-9.

5. Tang F, Wang J, Tang Z, Kang M, Deng Q, Yu J. Accuracy of McMonnies Questionnaire as a Screening Tool for Chinese Ophthalmic Outpatients. Virgili G,ed.PLoS ONE. 2016;11(4):e0153047.

6. Nichols KK, Nichols JJ, Zadnik K. Frequency of dry eye diagnostic test procedures used in various modes of ophthalmic practice. Cornea 2000;19:477-82

7. Milner MS, Beckman KA, Luchs JI, et al. Dysfunctional tear syndrome: dry eye disease and associated tear film disorders - new strategies for diagnosis and treatment. Current Opinion in Ophthalmology. 2017;28(Suppl 1):3-47.

8. Bhatnagar KR, Pote S, Pujari S, Deka D. Validity of subjective assessment as screening tool for dry eye disease and its association with clinical tests. International Journal of Ophthalmology.2015;8(1):174-181.

9. Messmer EM. The Pathophysiology, Diagnosis, and Treatment of Dry Eye Disease.Deutsches Ärzteblatt International.2015;112(5):71-82.

10. Gothwal VK, Pesudovs K, Wright TA, MacMonnies CW. McMonnies questionnaire: enhancing screening for dry eye syndromes with Rasch analysis. Invest OphthalmolVis Sci. 2010;51(3):1401-1407.

11. Guo Y, Peng R, Feng K, Hong J. Diagnostic Performance of McMonnies Questionnaire as a Screening Survey for Dry Eye: A Multicenter Analysis. Journal of Ophthalmology. 2016;2016:6210853.

12. Khurana AK, Chaudhary R, Ahluwalia BK. A new criteria to diagnose and grade dry eye. India Ophthalmology Today 1993;71-3.

13. Basak SK, Pal PP, Basak S, Bandyopadhyay A, Choudhury S, Sar S. Prevalence of dry eye diseases in hospital-based population in West Bengal, Eastern India. Journal of the Indian Medical Association. 2012 Nov; 1 10(11):189-94.

14. Bhutia $\mathrm{KL}$, Lomi N. A hospital-based study to estimate the proportion of dry eye cases among patients attending the Tertiary Care Hospital, Gangtok, Sikkim Sudanese J Ophthalmol [serial online] 2016 [cited 2017 Jul 11]:8:42-5.

15. Gayton JL. Etiology, prevalence, and treatment of dry eye disease. Clinical Ophthalmology. 2009;3:405-412

16. Guillon M, Maïssa C. Tear film evaporation - effect of age and gender. Cont Lens Anterior Eye. 2010;33:171-5.

17. Schaumberg DA, Dana R, Buring JE, Sullivan DA. Prevalence of dry eye disease among US men: Estimates from the Physicians' Health Studies. Arch Ophthalmol.2009; 127:763-8.

18. Moss SE, Klein R, Klein BE. Incidence of dry eye in an older population. Arch Ophthalmol.2004;122:369-73.

19. Abdelaziz MM, Fahim SA, Mousa DB, Gaya BI. Effects of computer use on visual acuity and colour vision. Eur J Sci Res. 2009;35:99-105.

20. Sahai A, Malik P. Dry Eye: Prevalence and Attributable Risk Factors in a Hospital-Based Population. Indian J Ophthalmol 2005;53:87-91

21. Garg A, Sheppard JD, Donnenfeld ED, Meyer D, Cyres MK, editors. Clinical Diagnosis and Management of Dry Eye and Ocular Surface Disorders. $1^{\text {st }} \mathrm{ed}$, Ch.3\& 5.India:Jaypee publisher;2006.p. 49-50,69-70.

22. Sahai A, Malik P. Dry Eye: Prevalence and attributable risk Factors in a Hospital-Based Population. Indian J Ophthalmol 2005;53:87-91.

23. Shah S, Jani H. Prevalence and associated factors of dry eye: Our experience in patients above 40 years of age at a Tertiary Care Center. Oman Journal of Ophthalmology.2015;8(3):151-156.

24. Choudhary P, Chalisgaonkar C, Lakhtakia S, Dwivedi A, Kain S. Dry eye prevalence and attributable risk factors in the eastern Madhya Pradesh. Int J Med Sci Public Health 2015:4:1556-1560

25. Bhanderi DJ, Choudhary S, DoshiVG. A community-based study of asthenopia in computer operators. Indian J Ophthalmol. 2008;56:51-55. 